\title{
ON ASYMPTOTIC ISOPERIMETRIC CONSTANT OF TORI
}

\author{
D. Burago And S. Ivanov
}

In this note we continue the study of asymptotic invariants of Riemannian tori (e.g. see $[\mathrm{BuI}]$ and references there). By asymptotic invariants we mean invariants which do not change under passing to finite covers. In [BuI] we show that the asymptotic volume growth of a Riemannian torus is at least as fast as that of a flat one. One may ask what are the possible values of "asymptotic isoperimetric constants" for such metrics (see definition below). We show that the asymptotic isoperimetric constant of a conformally flat torus is no less than that of a flat one, while for general metrics (in dimensions higher than 2) this constant may be arbitrarily small.

Let $(M, g)$ be the universal cover of a Riemannian $n$-torus.

Definition. We define the asymptotic isoperimetric constant $\sigma(M, g)$ of $(M, g)$ by

$$
\sigma(M, g)=\limsup _{\operatorname{Vol}_{n}(\Omega) \rightarrow \infty} \frac{\operatorname{Vol}_{n}(\Omega, g)^{1 / n}}{\operatorname{Vol}_{n-1}(\partial \Omega, g)^{1 / n-1}}
$$

where $\mathrm{Vol}_{n}$ and $\mathrm{Vol}_{n-1}$ are Riemannian measures (for $g$ ) of the respective dimensions and $\Omega$ ranges over all open bounded subsets of $M$.

Clearly $\sigma(M, g)$ is finite, positive, and invariant under homotheties of the metric.

We denote by $\sigma_{n}$ the isoperimetric constant of the standard Euclidean $n$-space,

$$
\sigma_{n}=\frac{m_{n}\left(D^{n}\right)^{1 / n}}{m_{n-1}\left(S^{n-1}\right)^{1 /(n-1)}},
$$

where $m_{n}$ and $m_{n-1}$ are the standard (Euclidean) measures of the respective dimensions.

The proof of the following theorem is to some extent motivated by the Besikowitch counter-example to Loewner's conjecture, see [Bes]. (Loewner conjectured that if on a cylinder $D^{2} \times[0,1]$ we are given a Riemannian metric such that the distance between the foundations $D^{2} \times 0$ and $D^{2} \times 1$ is no less than $h$ and the area of each "section" is of area no less than $l$, then the volume $\left.\operatorname{Vol}\left(D^{2} \times[0,1]\right) \geq h l\right)$. Notice that, although the metric in the Besikowitch counter-example is conformally flat, the Theorem does not hold for conformally flat metrics (see Proposition).

The first author is partially supported by a Sloan Foundation Fellowship and NSF grant DMS95-05157. The second author is partially supported by RFFR grant 96-01-00676 and CRDF grant RM1-169.

Typeset by $\mathcal{A}_{\mathcal{M S}}-\mathrm{T}_{\mathrm{EX}}$ 
Theorem. If $n \geq 3$, then there exist $\mathbf{Z}^{n}$-periodic Riemannian metrics on $\mathbf{R}^{n}$ with arbitrarily small asymptotic isoperimetric constants.

Proof. Let $\operatorname{pr}_{i}: \mathbf{R}^{n} \rightarrow \mathbf{R}^{n-1}$ denote the projection onto the $i$ th coordinate hyperplane. Fix $n$ congruent open balls $U_{1}, \ldots U_{n}$ in $I^{n-1}$ (where $I=[0,1]$ ), such that the "tubes" $G_{i}:=\operatorname{pr}_{i}^{-1}\left(U_{i}\right)$ in $\mathbf{R}^{n}$ are disjoint. This is possible since $n \geq 3$. Let $v=m_{n-1}\left(U_{1}\right)=\cdots=m_{n-1}\left(U_{n}\right)$. Now pick a small $\varepsilon>0$ and construct a $\mathbf{Z}^{n}$-periodic Riemannian metric $g_{\varepsilon}$ on $\mathbf{R}^{n}$ such that

(1) The volume form determined by $g_{\varepsilon}$ is equal to $m_{n}$.

(2) In every tube $G_{j}, j=1, \ldots, n$, the line element of $g_{\varepsilon}$ is given by

$$
d s^{2}=\varepsilon^{2 n-2} d x_{j}^{2}+\sum_{i \neq j} \frac{d x_{i}^{2}}{\varepsilon^{2}} .
$$

We will prove that $\sigma\left(\mathbf{R}^{n}, g_{\varepsilon}\right) \rightarrow 0$ as $\varepsilon \rightarrow 0$. Consider a domain $\Omega \subset \mathbf{R}^{n}$ and let $V=\operatorname{Vol}_{m}\left(\Omega, g_{\varepsilon}\right)=m_{n}(\Omega)$. We think of $\mathbf{R}^{n}$ as the union of "cells" $I^{n}+k, k \in \mathbf{Z}^{n}$, each cell carrying a copy of the same metric. Let $\Omega^{\prime}$ denote the union of cells $I^{n}+k$ for which $m_{n}\left(\Omega \cap\left(I^{n}+k\right)\right)>1-v / 2$. To complete the proof, we will derive a lower bound for $\operatorname{Vol}_{n-1}\left(\partial \Omega, g_{\varepsilon}\right)$ in terms of $V$ and $\varepsilon$. We argue separately for the two following cases.

Case 1: $m_{n}\left(\Omega^{\prime}\right) \geq V / 2$. By a known Loomis-Whitney inequality [LW] the volume of $\Omega^{\prime}$ can be estimated from above in terms of volumes of its projections:

$$
\prod_{i=1}^{n} m_{n-1}\left(\operatorname{pr}_{i}\left(\Omega^{\prime}\right)\right) \geq m_{n}\left(\Omega^{\prime}\right)^{n-1} .
$$

Hence there exists $j, 1 \leq j \leq n$, for which $m_{n-1}\left(\operatorname{pr}_{j}\left(\Omega^{\prime}\right)\right) \geq m_{n}\left(\Omega^{\prime}\right)^{1-1 / n}$. The projection $\operatorname{pr}_{j}\left(\Omega^{\prime}\right)$ is a union of $(n-1)$-dimensional cells of the form $I^{n-1}+k$, $k \in \mathbf{Z}^{n-1}$. For every cell $I^{n-1}+k \subset \operatorname{pr}_{j}\left(\Omega^{\prime}\right)$ we have

$$
m_{n-1}\left(\operatorname{pr}_{j}(\Omega) \cap\left(I^{n-1}+k\right)\right)>1-v / 2
$$

and therefore

$$
m_{n-1}\left(\operatorname{pr}_{j}(\Omega) \cap\left(U_{j}+k\right)\right)>v / 2 .
$$

Note that $\operatorname{pr}_{j}(\partial \Omega)=\operatorname{pr}_{j}(\Omega)$ since $\Omega$ is bounded. The definition of $g_{\varepsilon}$ implies that $m_{n-1}\left(\operatorname{pr}_{j}(X)\right) \leq \varepsilon^{n-1} \operatorname{Vol}_{n-1}\left(X, g_{\varepsilon}\right)$ for any set $X \subset \operatorname{pr}_{j}^{-1}\left(U_{j}+k\right)$, so

$$
\operatorname{Vol}_{n-1}\left(\partial \Omega \cap \operatorname{pr}_{j}^{-1}\left(U_{j}+k\right), g_{\varepsilon}\right) \geq \frac{v}{2} \cdot \varepsilon^{-(n-1)} .
$$

Therefore

$$
\operatorname{Vol}_{n-1}\left(\partial \Omega, g_{\varepsilon}\right) \geq \frac{v}{2} \cdot \varepsilon^{-(n-1)} \cdot m_{n-1}\left(\operatorname{pr}_{j}\left(\Omega^{\prime}\right)\right) \geq c(n) \cdot \varepsilon^{-(n-1)} \cdot m_{n}\left(\Omega^{\prime}\right)^{1-1 / n}
$$

Replacing $m_{n}\left(\Omega^{\prime}\right)$ by $V$ in the last expression will only affect the constant.

Case 2: $m_{n}\left(\Omega^{\prime}\right)<V / 2$. Consider the cells of the form $I^{n}+k, k \in \mathbf{Z}^{n}$, intersecting with $\Omega$ with $m_{n}\left(\Omega \cap\left(I^{n}+k\right)\right) \leq 1-v / 2$. It is easy to check (e.g., by applying the 
Loomis-Whitney inequality ([LW]) to the set $\Omega \cap \operatorname{Int}\left(I^{n}+k\right)$ ) that, for these cells, the values

$$
\frac{m_{n-1}\left(\partial \Omega \cap \operatorname{Int}\left(I^{n}+k\right)\right)}{m_{n}\left(\Omega \cap \operatorname{Int}\left(I^{n}+k\right)\right)}
$$

have a positive lower bound depending only on $v$ and the dimension $n$. Since the terms $m_{n}\left(\Omega \cap \operatorname{Int}\left(I^{n}+k\right)\right)$ adds up to a value of at least $m_{n}\left(\Omega \backslash \Omega^{\prime}\right) \geq V / 2$, this gives us the following estimation

$$
\operatorname{Vol}_{n-1}\left(\partial \Omega, g_{\varepsilon}\right) \geq c(n, \varepsilon) \cdot V
$$

for case 2 .

As $V \rightarrow \infty$, the inequalities obtained for the two cases take the form

$$
\limsup \frac{V^{1 / n}}{\operatorname{Vol}_{n-1}\left(\partial \Omega, g_{\varepsilon}\right)^{1 /(n-1)}} \leq c(n) \cdot \varepsilon
$$

and

$$
\limsup \frac{V^{1 / n}}{\operatorname{Vol}_{n-1}\left(\partial \Omega, g_{\varepsilon}\right)^{1 /(n-1)}}=0
$$

respectively. Thus we obtain that $\sigma\left(\mathbf{R}^{n}, g_{\varepsilon}\right) \leq c(n) \cdot \varepsilon$.

Proposition. If $(M, g)$ is a universal cover of a conformally flat torus, then $\sigma(M, g) \geq$ $\sigma_{n}$, and the equality holds if and only if the metric is flat.

Proof. Our argument is an application of the so-called length-area method and is similar to Loewner's proof of the 2-dimensional isosystolic inequality (see $[\mathrm{G}]$, page 4). Let $M=\mathbf{R}^{n}$, and let the metric $g$ be given by $g=\lambda g_{E}$ where $g_{E}$ is the standard Euclidean metric, $\lambda$ is a positive smooth function on $\mathbf{R}^{n}, \lambda$ is periodic with respect to some (co-compact) lattice $\Gamma \subset \mathbf{R}^{n}$. Without loss of generality we may assume that the volume of $\mathbf{R}^{n} / \Gamma$ is equal to 1 for both metrics $g$ and $g_{E}$, i.e.

$$
\int_{\mathbf{R}^{n} / \Gamma} \lambda^{n} d m_{n}=m_{n}\left(\mathbf{R}^{n} / \Gamma\right)=1 .
$$

Define $\alpha:=\int_{\mathbf{R}^{n} / \Gamma} \lambda^{n-1} d m_{n}$. By Hölder inequality, the above identity implies that $\alpha<1$ unless $\lambda \equiv 1$.

For $x \in \mathbf{R}^{n}$ and $r>0$, denote by $B(x, r)$ the Euclidean ball of radius $r$ centered at $x$. To prove that $\sigma(M, g) \geq \sigma_{n}$ it suffices to construct a sequence of regions $\Omega_{i}$ with volumes growing to infinity and

$$
\lim _{i \rightarrow \infty} \frac{\operatorname{Vol}_{n}\left(\Omega_{i}, g\right)^{1 / n}}{\operatorname{Vol}_{n-1}\left(\partial \Omega_{i}, g\right)^{1 / n-1}} \geq \sigma_{n} .
$$

We will show that such regions can be chosen among Euclidean balls $B(x, r)$.

It is clear that

$$
\frac{\operatorname{Vol}_{n}(B(x, r), g)}{m_{n}(B(x, r))} \rightarrow 1 \quad \text { as } r \rightarrow \infty,
$$

with the convergence being uniform in $x$. (Indeed, both $\operatorname{Vol}(B(x, r), g)$ and $m_{n}(B(x, r)$ ) are asymptotically equal to the number of fundamental domains of $\Gamma$ contained in $B(x, r))$. Define

$$
A_{r}(x)=\frac{\operatorname{Vol}_{n-1}(\partial B(x, r), g)}{m_{n-1}(\partial B(x, r))},
$$


then $A_{r}(x)$ is the average value of the function $\lambda^{n-1}$ over the Euclidean sphere $\partial B(x, r)$. Thus the function $A_{r}$ is $\Gamma$-periodic. A standard argument of Euclidean integral geometry shows that the average value of $A_{r}$ (w.r.t. the standard Euclidean volume $\left.m_{n}\right)$ is equal to $\alpha$. Therefore for every $r>0$ there is a a point $x_{0}=x_{0}(r) \in$ $\mathbf{R}^{n}$ such that $A_{r}\left(x_{0}\right) \leq \alpha$. To complete the proof, observe that

$$
\begin{aligned}
\sigma(M, g) & \geq \limsup _{r \rightarrow \infty} \frac{\operatorname{Vol}_{n}\left(B\left(x_{0}(r)\right), g\right)^{1 / n}}{A_{r}\left(x_{0}(r)\right)^{1 /(n-1)}} \\
& \geq \frac{1}{\alpha^{1 /(n-1)}} \cdot \frac{m_{n}\left(B\left(x_{0}(r)\right)\right)^{1 / n}}{m_{n-1}\left(\partial B\left(x_{0}(r)\right)\right)^{1 /(n-1)}}=\frac{\sigma_{n}}{\alpha^{1 /(n-1)}} .
\end{aligned}
$$

Remark. The Proposition implies, in particular, that the asymptotic isoperimetric constant of an arbitrary 2-dimensional torus is at least $\sigma_{2}$, since all 2-tori are conformally flat. There is a direct 2-dimensional argument, which may give better geometric insight and suggests different candidates for "optimal" regions $\Omega_{i}$. We give an outline of the argument below.

In the notations of the Proposition, denote by $E_{i}$ the ellipse of maximum area inscribed in the ball of radius $i$ in the stable norm of $g$ (see [BuI] for definitions). The proof of the volume growth theorem [BuI] implies that the area enclosed in $E_{r}$ in $g$ is at least $\pi i^{2}+o\left(i^{2}\right)$. Choose $\sqrt{i}$ points on $E_{i}$ such that all distances between neighboring points are equal, an let $\Omega_{i}$ be the interior of a geodesic polygon (w.r.t. $g$ ) with these vertices. One easily sees that $\lim _{i \rightarrow \infty} \frac{\operatorname{Vol}_{2}\left(\Omega_{i}\right)}{\operatorname{Vol}_{2}\left(E_{i}\right)}=1$. On the other hand, the length of each side of $\Omega_{i}$ differs from the distance between its endpoints in the stable norm by no more than a constant $c$. This distance, in its turn, is no greater than the distance between these endpoints in the Euclidean metric for which $E_{i}$ is a ball of radius $i$. Combining these inequalities, one concludes that $\operatorname{Vol}_{1}\left(\partial \Omega_{i}\right) \leq 2 \pi i+c \sqrt{i}$, that completes the argument.

Remark. Reasoning as above, one easily sees that, for given asymptotic volume growth, in dimension 2 the exact value of $\sigma(M, g)$ can be recovered from the stable norm (this observation was made independently by P. Pansu.) This is no longer true in higher dimensions, as one can observe from the examples in the proof of the theorem.

Acknowledgment. We would like to thank Yu. Burago who has drawn our attention to the Besikowitch counter-example to Loewner's conjecture. The first author would also like to thank the Erwin Schroedinger International Institute for Mathematical Physics for the opportunity to spend two weeks at the Institute.

\section{REFERENCES}

[Bes] A. S. Besikowitch, On two problems of Loewner, Mat. Zametki 27 (1952), 141-144.

[BuI] D. Burago, S. Ivanov, On asymptotic volume of tori, GAFA 5 (1995), no. 5, 800-808.

[G] M. Gromov, Filling Riemannian manifolds, J. Diff. Geom. 18 (1983), 1-147.

[LW] L. Loomis, H. Whitney, An inequality related to the isoperimetric inequality, Bull. AMS 55 (1949), no. 10, 961-962.

Dmitri Burago, PennState University, 228 McAllister Bld., University Park, PA $16802-6403$

E-mail address: burago@math.psu.edu

Sergei Ivanov, Steklov Institute of Mathematics, 191011, Fontanka 27, St. PeTERSBURG, RUSSIA

E-mail address: svivanov@pdmi.ras.ru 